\title{
DISEÑO DIGITAL E IMPRESIÓN 3D DE ESTRUCTURAS SUPERFICIALES DE GRANDES LUCES
}

Eje 1: Innovación en sistemas constructivos/estructurales

\author{
Boccolini Vanina ${ }^{1}$ \\ Prat Emma Susana² \\ Vedoya Daniel Edgardo 3 \\ Ramirez Gisela Natalia ${ }^{4}$ \\ ${ }^{1}$ Facultad de Arquitectura y Urbanismo, UNNE, Argentina, vnboccolini@hotmail.com \\ 2 Facultad de Arquitectura y Urbanismo, UNNE, Argentina, emmasus@hotmail.com \\ ${ }^{3}$ Facultad de Arquitectura y Urbanismo, UNNE, Argentina, devedoya@gmail.com \\ ${ }^{4}$ Facultad de Arquitectura y Urbanismo, UNNE, Argentina, gise_ram03@hotmail.com
}

\section{RESUMEN}

El Seminario Taller de Análisis y Diseño Estructural (E-III_EnLinea) está destinado a los alumnos del $5^{\circ}$ año de la carrera de Arquitectura y Urbanismo de la Facultad de Arquitectura de la Universidad Nacional del Nordeste. El mismo plantea una visión alternativa del diseño estructural y arquitectónico enfatizando la necesidad del análisis de las estructuras no tradicionales de grandes luces desde el inicio del proceso de aprendizaje.

Durante el ciclo lectivo 2017 se propuso la realización de un TCF (Trabajo de Campo Final) que implicó el diseño de estructuras de grandes luces con tecnologías constructivas especiales articulando su materialización con tecnología de impresión 3D. La temática abordada correspondía a la Unidad Didáctica 4 de la asignatura: Tipos estructurales conformados con componentes superficiales rígidos, Estructuras de Curvatura Total Nula, Positiva y Negativa.

Los objetivos de la experiencia se diseñaron buscando: reforzar los conocimientos relativos al comportamiento estructural de estructuras de grandes luces conformadas por componentes superficiales rígidos; experimentar con tecnologías constructivas alternativas del tipo de la Impresión 3D y tipos de ensayos aplicables a modelos analógicos y digitales; incorporar metodologías de trabajo colaborativo, interdisciplinario, presencial y virtual a las actividades universitarias y futuras prácticas profesionales; generar un espacio de trabajo colaborativo con otras áreas disciplinares.

EI TCF se organizó en tres etapas. Primeramente, se centró en la definición morfológica en 3D de modelos digitales de estructuras básicas del tipo de cáscaras; a continuación, se trabajó en la 
capacitación de los alumnos en la temática relativa a la impresión 3D para concluir esta etapa con la impresión 3D de los modelos; finalmente se definieron los tipos de ensayos mecánicos analógicos y digitales.

El trabajo implementó metodologías de trabajo colaborativo, interterdisciplinario, presencial y virtual, involucrando distintos espacios académicos de la UNNE para lo cual se gestionó un acuerdo específico de cooperación y asistencia técnica entre la cátedra Estructuras 3 y el CEDIA (Centro de Desarrollo de Informática Aplicada) de la Facultad de Arquitectura, el Laboratorio de Diseño e Impresión 3D y el INTECNE de la Facultad de Ingeniería, todos espacios institucionales de la UNNE.

Cada uno de los objetivos fue alcanzado satisfactoriamente logrando dar inicio y fin a la propuesta en tiempo y forma, con la colaboración y motivación constante de todos los docentes y estudiantes involucrados, y la formulación final de interesantes conclusiones y generación de nuevas propuestas a través de los resultados alcanzados en cada una de las etapas.

\section{PALABRAS CLAVES: DISEÑO DIGITAL - IMPRESIÓN 3D - ESTRUCTURAS DE GRANDES LUCES - TECNOEDUCACIÓN}

\section{INTRODUCCIÓN}

El Seminario Taller de Análisis y Diseño Estructural (E-III_EnLinea) es un espacio curricular destinado a los alumnos del $5^{\circ}$ año de la carrera de Arquitectura y Urbanismo de la Facultad de Arquitectura y Urbanismo de la Universidad Nacional del Nordeste (FAU-UNNE). El mismo plantea una visión alternativa del diseño estructural y arquitectónico enfatizando la necesidad del análisis de las estructuras no tradicionales de grandes luces desde el inicio del proceso de aprendizaje.

Durante el ciclo lectivo 2017 se propuso la realización de un TCF (trabajo de campo final) cuya temática se centró en el diseño estructural de grandes luces con tecnologías constructivas especiales y la materialización de los modelos a través de la impresión 3D, articulando de esta forma ejercicios académicos con prácticas profesionales reales, actuales y con proyección laboral a largo plazo.

Los contenidos abordados correspondían a la Unidad Didáctica 4 de la Asignatura denominada Tipos estructurales conformados con componentes superficiales rígidos, Estructuras de Curvatura Total Nula, Positiva y Negativa.

Para ello se implementaron metodologías de participación activa centradas en el alumno y en los grupos, tanto presenciales como virtuales y de trabajo colaborativo e interdisciplinario involucrando a: la cátedra y el CEDIA (Centro de Desarrollo de Informática Aplicada) de la Facultad de Arquitectura, sumados al Laboratorio de Diseño e Impresión 3D y el Laboratorio de Aerodinámica ambos de la Facultad de Ingeniería, todos espacios institucionales de la UNNE.

Indudablemente las metodologías propuestas buscaron motivar en el estudiante el desarrollo de habilidades cognitivas superiores que, tal como expresa Raths, Wassermann y otros (1971, pág. 60) propician "...sin duda un tipo de estudiante que será más cauto y sagaz al abrir juicios y sacar conclusiones. Estudiantes que verán más de un derrotero de acción... que buscarán alternativas y serán receptivos para las suposiciones... que guardarán el tesoro de la duda. Serán mentalmente 
más abiertos y quizás estarán mejor preparados para cambiar en múltiples niveles y esferas de actividades. Probablemente su visión de la vida será más rica en experiencia y en lugar de mostrarse resistentes a enfrentar los problemas, los abordarán con entereza y energía... Cuanto mejor sea la base, con tanta mayor rapidez podremos anticipar el florecimiento de un estudio inteligente comprensivo del hombre y de sus problemas. Subrayar la importancia del pensamiento implica dar un gran paso inicial para el mejoramiento de la condición humana". ${ }^{1}$

\section{DESARROLLO}

Cabe destacar que el diseño del TCF requirió de varias tareas antes, durante y después de su concreción.

a. Gestión. Inicialmente fue necesaria la gestión de acuerdos de colaboración entre disciplinas, definiendo claramente el alcance de las tareas a cargo de cada parte.

Para ello se invitó a miembros de la Facultad de Ingeniería a aportar sus conocimientos, docentes del Laboratorio de Diseño e Impresión 3D y del Túnel del Viento del Laboratorio de Aerodinámica:

- los primeros dedicados a trabajar específicamente en la fase de impresión en 3 dimensiones de los modelos digitales diseñados por los alumnos, colaborando no solamente con las clases de formación en el uso de la tecnología de impresión 3D sino también aportando el equipamiento e insumo necesario (impresoras y material plástico), y

- los segundos implicados en los ensayos mecánicos y digitales a realizarse sobre los modelos impresos.

Las actividades entre equipos académicos se formalizaron a través de la firma de un Acuerdo Específico de Colaboración con duración de 2 (dos) años o ciclos lectivos.

b. Objetivos planteados:

- Reforzar los conocimientos relativos al comportamiento estructural de estructuras de grandes luces conformadas por componentes superficiales rígidos analizados durante el cursado.

- Motivar la adquisición de destrezas tecnológicas, tanto digitales como analógicas, en procesos de materialización de objetos y espacios diseñados experimentando tecnologías de diseño y constructivas alternativas.

- Motivar en la toma de decisiones para resolver problemas articulados con la futura práctica profesional.

- Promover metodologías de trabajo colaborativo, interdisciplinario, presencial y virtual en las actividades académicas motivando el desarrollo de destreza comunicacionales.

- Reforzar capacidades relacionadas al pensamiento crítico, el aprendizaje autodirigido, así como habilidades de evaluación y autoevaluación.

\footnotetext{
${ }^{1}$ Si bien el autor hace hincapié en iniciar con este tipo de formación desde el nivel inicial, siempre hay tiempo de ofrecer a nuestros estudiantes la oportunidad de alcanzar dichos conocimientos, habilidades y destrezas, considerando además disciplinas del tipo de la Arquitectura cuyo abanico de aplicaciones laborales es cultural y socialmente muy amplio.
} 


\section{c. Metodología o Estrategias Pedagógicas.}

En base a los objetivos formulados y dada la naturaleza del proyecto, se buscó aplicar estrategias pedagógicas de participación activa centradas en el alumno, buscando reforzar no solo contenido teórico y práctico relativo a la temática abordada, sino también motivar la toma de decisiones, individuales y grupales, en la resolución de problemas articulados a la verdadera práctica profesional, así como reforzar capacidades relacionadas al pensamiento crítico, el aprendizaje autodirigido, así como habilidades de evaluación y autoevaluación.

Para ello se adoptó principalmente el Enfoque de Aprendizaje Basado en Problemas o ABP complementado con metodologías de trabajo colaborativo y metacognición, y en menor porcentaje por el método directo o clase tradicional.

Tal como afirma Agustina Blanco (2018) "el foco está puesto en el alumno como protagonista... el docente pasa a ser una guía... es imposible pensar en una sociedad pasiva y observadora y que el pensamiento lineal ya no da respuestas..."2

Desde esta perspectiva el ABP no es solo una técnica, es todo un enfoque como metodología con, básicamente, tres etapas o procesos claros: "planificación, desarrollo y evaluación con indagación constante. Se puede enfocar en el trabajo entre disciplinas y desde allí desarrollar un trabajo, o directamente plantear un tema". En todos los casos el alumno es el protagonista y la interdisciplinariedad atraviesa todas las fases del proceso. El estudiante planifica con contenidos y metas en vistas a la problemática a resolver. Se presentan estándares e indicadores en base a capacidades, que les permite ir evaluando sus propios avances, lo que va ocurriendo.

c. Desarrollo. De acuerdo a los objetivos y estrategias establecidas el TCF se desarrolló en 2 (dos) etapas.

\section{$1^{\circ}$. ETAPA 1. Definición Morfológica del Modelo Digital e Impresión 3D.}

Realizada durante el mes de noviembre de 2017, consistió en:

- Definición de grupos de trabajo: de 2 a 4 integrantes por equipo.

- Capacitación práctica sobre Modelado Digital destinado a la Impresión 3D, a cargo de docentes del Laboratorio de Diseño e Impresión 3D de la Facultad de Ingeniería y del CEDIA (Centro de Desarrollo de Informática Aplicada) de la Facultad de Arquitectura (FAU)

- Tutorías teórico/prácticas, presenciales y virtuales de refuerzo referidas a la temática de diseño de Estructuras de Grandes Luces y de modelado analógico y digital a cargo de docentes del Seminario de Taller de Análisis y Diseño Estructural.

- Tutorías presenciales y virtuales en instancias de diseño del proyecto arquitectónico a materializar a cargo de docentes del Seminario de Taller de Análisis y Diseño Estructural.

- Diseño del proyecto arquitectónico: selección del tipo estructural que trabajaría cada equipo, diseño morfológico en 3D de los proyectos y modelado digital de los mismos.

${ }^{2}$ Entrevista en el espacio Conversaciones en La Nación, a Agustina Blanco ex directora ejecutiva de la ONG Educar 2050.

http://corrientesmagazine.com/2018/01/27/lo-que-viene-en-educacion-que-es-el-aprendizaje-basado-en-proyectos/ 27 de enero de 2018. 
- Materialización en 3 dimensiones de todos los modelos digitales diseñados por cada equipo.

- Presentación final de los trabajos realizados conteniendo: memoria descriptiva digital multimedial con desarrollo de la propuesta (formatos doc, pdf, ppt, exe de prezi), archivos de los diseños tridimensionales (dwg, skp, stl) y los modelos impresos en 3D.

\section{$2^{\circ}$. ETAPA 2. Ensayo de los modelos impresos.}

Finalizada la primera etapa, se procedió a iniciar el desarrollo de la segunda a partir de marzo de 2018. Al momento de realización del presente artículo, esta etapa no ha sido concluida, quedando prevista su finalización para el segundo cuatrimestre del presente ciclo lectivo 2018.

- Discusión de resultados y selección de los modelos impresos más aptos para someter ensayo.

- Realización de los ensayos mecánicos, analógicos y digitales previstos en el proyecto

- Generación de informes parciales y finales de procedimientos, resultados obtenidos y conclusiones.

d. Entornos y tecnologías. Ambas etapas involucraron múltiples espacios físicos y virtuales que enriquecieron el entorno y la colaboración entre los distintos actores interdisciplinarios involucrados: docentes, alumnos e investigadores de ambas carreras. Así como también se recurrió dispositivos tecnológicos analógicos y digitales que aportaron a la producción de las etapas del proceso de acuerdo a las necesidades presentadas.

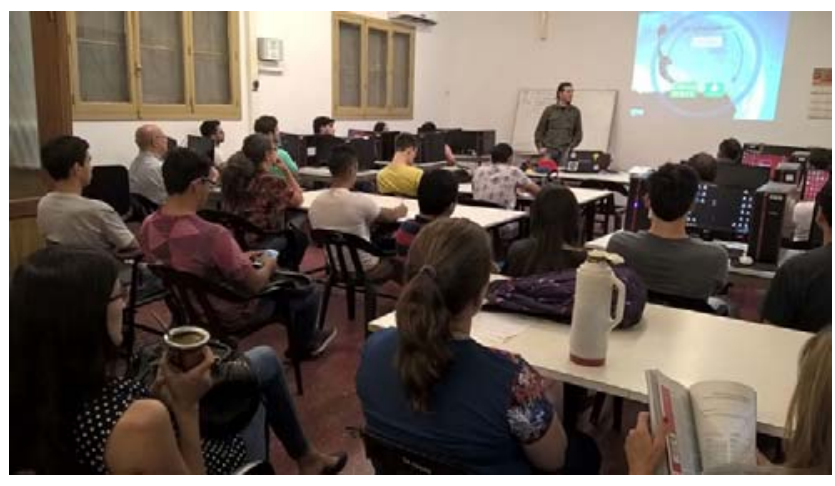

Fig. 1. Espacio: CEDIA, Fac. Arquitectura Actividad: Práctica en PC

Temática: Modelado Digital destinado a la Impresión 3D 


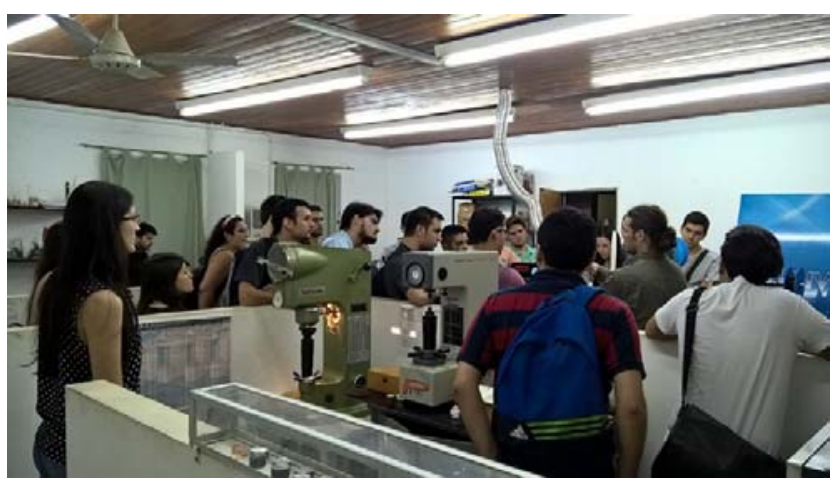

Fig. 2. Espacio: LAB. DISEÑO e IMPRESIÓN 3D, Fac. Ingeniería Actividad: Práctica en Impresoras 3D Temática: Impresión de modelos

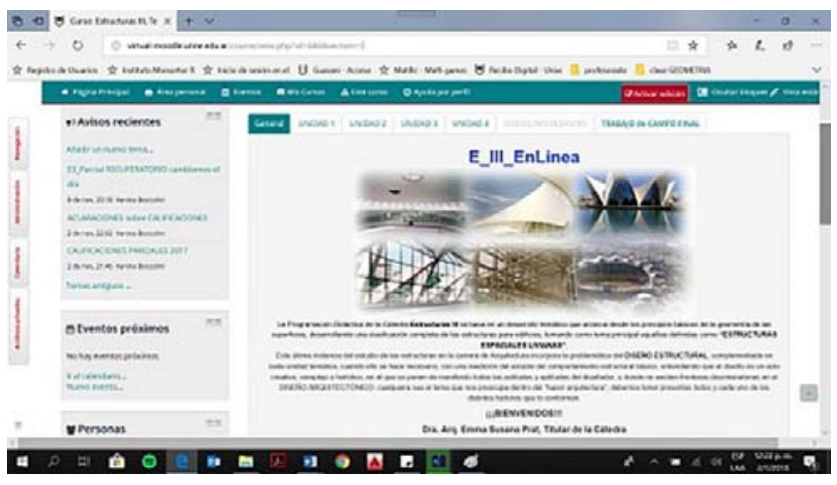

Fig. 3. Espacio: AULA VIRTUAL E-III_EnLinea Entorno MOODLE

Actividad: Tutorías y Prácticas Virtuales Temática: Contenido completo de la cátedra

La gestión de los entornos, así como los cronogramas de los encuentros, fueron previamente pautados entre docentes y alumnos a través de correo electrónico, aula virtual y/o red social. Los espacios compartidos fueron:

- Las tutorías presenciales se llevaron a cabo en la sede de la cátedra en el ITDAHu (Instituto de Investigaciones Tecnológicas para el Diseño Ambiental del Hábitat Humano)

- Las tutorías virtuales, sincrónicas o asincrónicas, se realizaron mediante aula virtual, red social y/o videochat.

- Las clases prácticas se desarrollaron presencialmente en el CEDIA de la FAU con visitas al Laboratorio de Diseño e Impresión 3D de Ingeniería.

- Las tareas de impresión 3D se realizaron Laboratorio de Diseño e Impresión 3D de Ingeniería.

Los dispositivos y herramientas tecnológicas digitales de producción utilizados de acuerdo a cada actividad fueron: 


\section{ARQUITECNO}

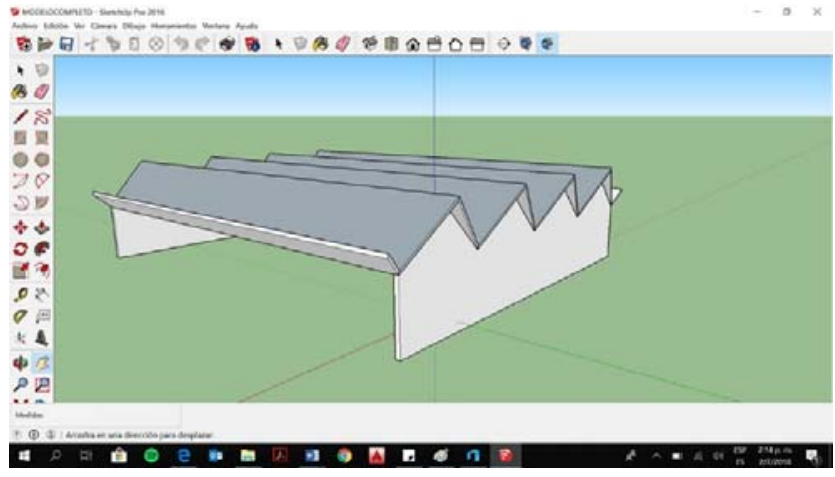

Fig.4. Actividad: diseño tridimensional de los proyectos arquitectónicos Hardware: PC _ Software: Sketchup / Autocad

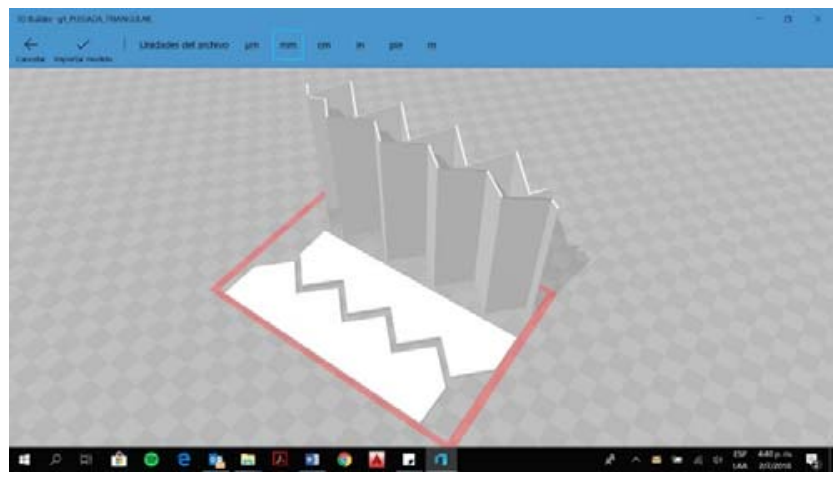

Fig. 5. Actividad: conversión de archivos dwg/skp a protocolo GCode Hardware: PC_Software: 3D Builder/Slic3r
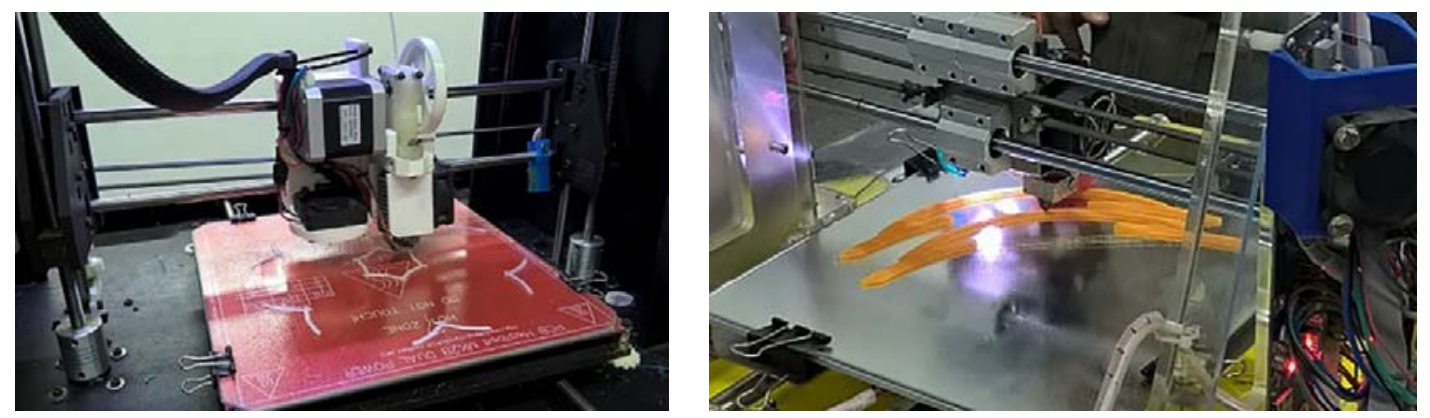

Fig. 6 y 7. Actividad: Impresión en 3 dimensiones de los proyectos Hardware: Impresoras 3D con material PLA (filamentos plásticos color) Software: Slic3r / Cura / Simplify

Además de los dispositivos digitales con fines productivos, específicamente para el diseño y materialización de los modelos) se utilizaron otros destinados a la capacitación, comunicación, colaboración y representación de las exposiciones parciales y finales de las creaciones. Entre ellos: herramientas del aula virtual Moodle (tareas, foros, material didáctico, etc.), correo electrónico, Red Social, VideoChat (Skype, Hangout, WhatsApp), Word, Power Point o Prezi, OneDrive de Microsoft y Google Drive, entre otros. 
e. Resultados. Más vale imágenes que palabras. A continuación, exponemos la producción de 3 (tres) grupos de un total de 7(siete) presentados y todos aprobados.
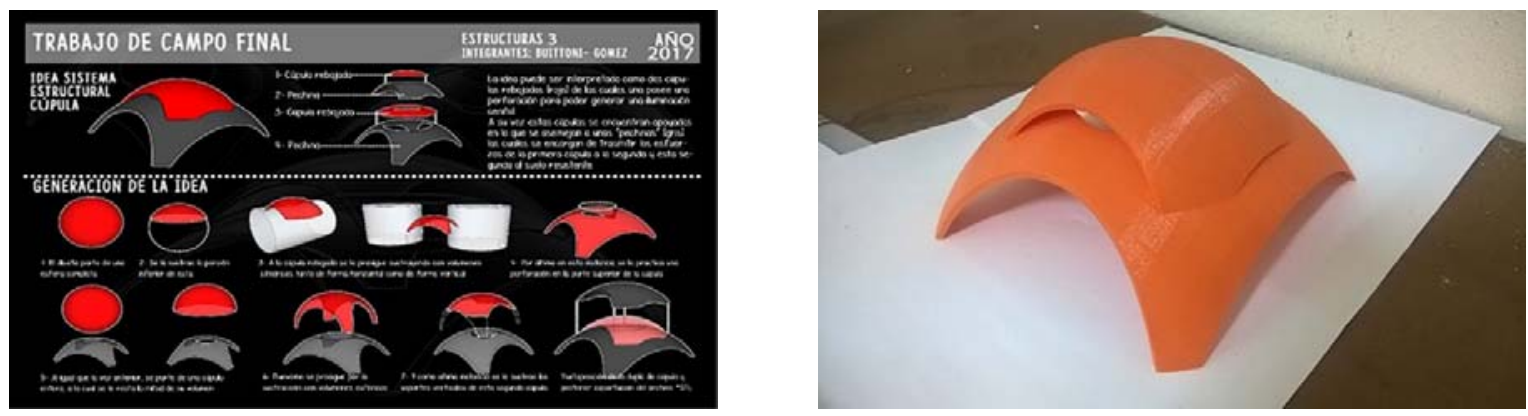

Fig. 8 y 9. Panel de Presentación y Modelo Impreso en 3D con PLA (filamentos plásticos)

Tipología: Cúpulas

Técnica constructiva: Cáscaras de Hormigón Armado
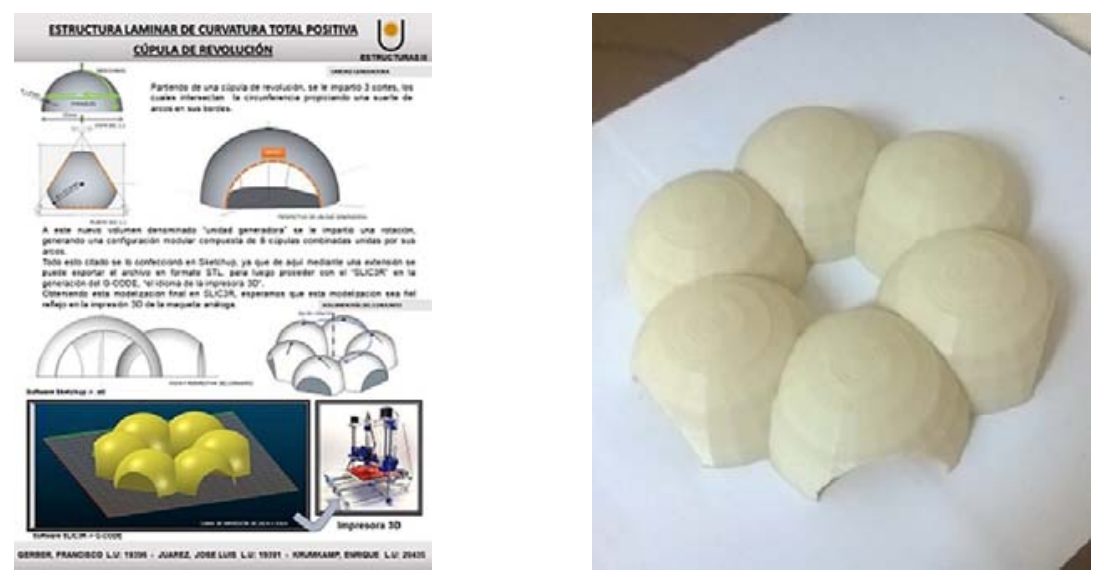

Fig. 10 y 11. Panel de Presentación y Modelo Impreso en 3D con PLA (filamentos plásticos)

Tipología: Cúpulas

Técnica constructiva: Cáscaras de Hormigón Armado
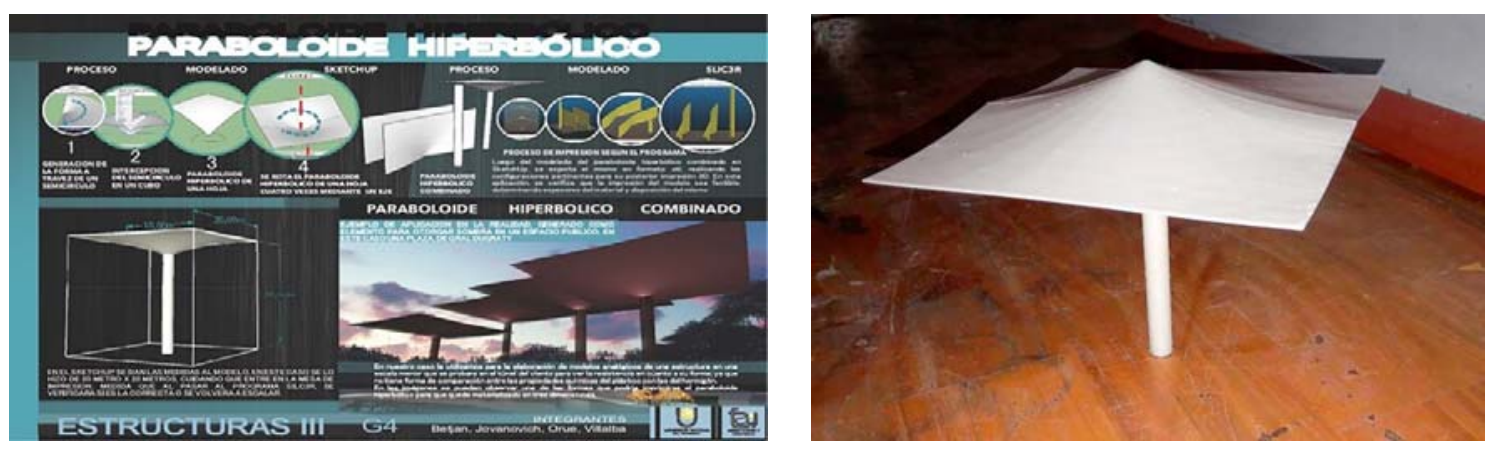

Fig. 12 y 13. Panel de Presentación y Modelo Impreso en 3D con PLA (filamentos plásticos)

Tipología: Paraboloide Hiperbólico

Técnica constructiva: Cáscaras de Hormigón Armado 


\section{CONCLUSIONES}

Los estudiantes no aprenden si no validan el conocimiento.

Tal afirmación nos impulsó a desarrollar el Trabajo de Campo Final (TCF) del Seminario Taller de Análisis y Diseño Estructural a través de las estrategias planteadas y consideramos, según los análisis del seguimiento durante el proceso, que los estudiantes han sido guiados en la búsqueda e incorporación de competencias tales como: la identificación de problemas relevantes del contexto profesional; la conciencia del propio aprendizaje; la planificación de las estrategias que se van a utilizar para aprender; el pensamiento crítico; el aprendizaje autodirigido; las habilidades de evaluación y autoevaluación; el aprendizaje permanente; la toma de decisiones; el trabajo en equipo; habilidades de comunicación (argumentación y presentación de la información), desarrollo de actitudes y valores, razonamiento eficaz y creatividad; búsqueda y manejo de información; investigación; entre otras.

Cabe destacar que las condiciones de planificación y desarrollo de la metodología ABP en el TCF están garantizadas en cuanto a los conocimientos previos de los alumnos, el contexto y el entorno de trabajo con acceso a distintas formas de comunicación (presencial y virtual) y tecnologías digitales acordes (conexión a internet, software y hardware apropiado), además de la capacidad de los docentes en la tarea de guía del proceso (generar disparadores no googleables) y la interdisciplina entre arquitectos, ingenieros y diseñadores.

Sin embargo, no se debe olvidar que más allá de todo esfuerzo y a similares condiciones curriculares:

...solo aprenderá el estudiante que esté dispuesto a hacerlo...

\section{BIBLIOGRAFÍA}

Conversaciones en La Nación (2018) Entrevista a Agustina Blanco. Buenos Aires (Argentina) Ed. Diario La Nación digital. http://corrientesmagazine.com/2018/01/27/lo-que-viene-en-educacionque-es-el-aprendizaje-basado-en-proyectos/

Dirección de Investigación y Desarrollo Educativo (2014) Las estrategias y técnicas didácticas en el rediseño. El Aprendizaje Basado en Problemas como técnica didáctica. Ed. Vicerrectoría Académica, Instituto Tecnológico y de Estudios Superiores de Monterrey. Monterrey (México)

Servicio de Innovación Educativa (UPM) de la Universidad Politécnica de Madrid (2008) Aprendizaje Basado en Problemas. Guías rápidas sobre nuevas metodologías. Ed. UPM. Madrid (España)

Solcoff, Karina (2016) Hacer memoria: aportes de la neuropsicología al aprendizaje. 1ra. Edición. Ed. Paidós Cuestiones de Educación. Ciudad Autónoma de Buenos Aires (Argentina)

Vedoya, D. E. y Prat, E. S. (2009) Estructuras de Grandes Luces. Tecnología y Diseño. Corrientes (Argentina) Ediciones del ITDAHu. 